Physics

Physics Research Publications

\title{
Hysteresis and noise from electronic nematicity in high-temperature superconductors
}
E. W. Carlson
K. A. Dahmen
E. Fradkin
S. A. Kivelson 


\title{
Hysteresis and Noise from Electronic Nematicity in High-Temperature Superconductors
}

\author{
E. W. Carlson, ${ }^{1}$ K. A. Dahmen, ${ }^{2}$ E. Fradkin, ${ }^{2}$ and S. A. Kivelson ${ }^{3,4}$ \\ ${ }^{1}$ Department of Physics, Purdue University, West Lafayette, Indiana 47907, USA \\ ${ }^{2}$ Department of Physics, University of Illinois, Urbana, Illinois 61801, USA \\ ${ }^{3}$ Department of Physics, Stanford University, Stanford, California 93105, USA \\ ${ }^{4}$ Department of Physics and Astronomy, University of California, Los Angeles, California 90095, USA
}

(Received 11 October 2005; published 7 March 2006)

\begin{abstract}
An electron nematic is a translationally invariant state which spontaneously breaks the discrete rotational symmetry of a host crystal. In a clean square lattice, the electron nematic has two preferred orientations, while dopant disorder favors one or the other orientations locally. In this way, the electron nematic in a host crystal maps to the random field Ising model. Since the electron nematic has anisotropic conductivity, we associate each Ising configuration with a resistor network and use what is known about the random field Ising model to predict new ways to test for local electronic nematic order (nematicity) using noise and hysteresis. In particular, we have uncovered a remarkably robust linear relation between the orientational order and the resistance anisotropy which holds over a wide range of circumstances.
\end{abstract}

In the high-temperature superconductors, in addition to superconductivity, there may exist various other types of order which break spatial symmetries of the underlying crystal, especially in the "pseudogap" regime at low doping [1-5]. However, it is often surprisingly difficult to obtain direct experimental evidence which permits one to clearly delineate in which materials, and in what range of temperature and doping, these phases occur. One such candidate order is the electronic nematic which breaks orientational, but not translational, symmetry [1]. Orientational long range order (LRO) induces transport anisotropy, so this is a natural way to look for nematic order $[1,6]$.

A nematic has two preferred orientations in a tetragonal crystal, so the order parameter can be represented by an Ising variable. On the other hand, since quenched disorder couples linearly to the nematic order parameter, an electron nematic in a square lattice with disorder maps to the random field Ising model (RFIM) [7] (see below). Interesting behavior reminiscent of the RFIM has been reported in recent noise [8] and hysteresis $[9,10]$ measurements on high-temperature superconductors. While in 3D, for sufficiently weak disorder, the RFIM exhibits a finite temperature phase transition to a low temperature ordered phase, in 2D the critical disorder strength is zero, and LRO is forbidden [11]. Even in the quasi-2D case of coupled RFIM planes, the critical disorder strength is exponentially small [12], so disorder will typically convert a true thermodynamic phase transition into a crossover. We focus here on two dimensions and demonstrate that even in this case, where orientational LRO is forbidden, it is possible to detect the proximity to order through noise and hysteresis measurements [8-10].

In a host crystal such as the cuprates, an electron nematic (which may arise, e.g., from local correlations of a melted stripe phase) tends to lock to favorable lattice directions, often either "vertically" or "horizontally" along $\mathrm{Cu}-\mathrm{O}$ bond directions. The two possible orientations can be represented by an Ising pseudospin $\sigma= \pm 1$ [13], and the tendency of neighboring nematic patches to align corresponds to a ferromagnetic interaction. In any given region, disorder due to dopant atoms between the cuprate planes produces electric field gradients which locally favor one orientation or the other and act like a random field on the electronic nematic, as illustrated in Fig. 1(a). Thus, the physics of an electron nematic is represented by the RFIM:

$$
H=-J \sum_{\langle i, j\rangle} \sigma_{i} \sigma_{j}-\sum_{i}\left(h+h_{i}\right) \sigma_{i}
$$

where $J>0$ is the coupling between neighboring nematic patches [14]. The local disorder field $h_{i}$ is taken to be Gaussian, with a disorder strength $\Delta$ characterized by the width of the Gaussian distribution. The symmetrybreaking field $h$ may be produced by, e.g., uniaxial strain, high current [15], magnetic field [9], or even orthorhom-

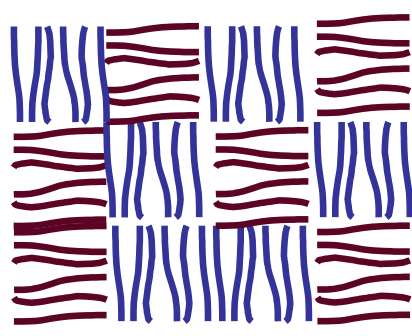

(a) Nematic patches

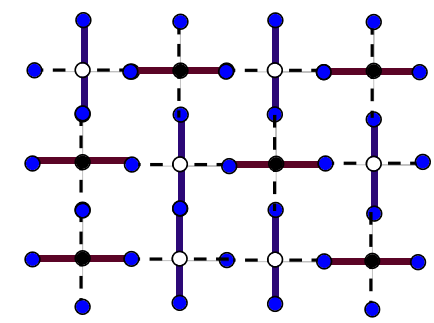

(b) Resistor Network
FIG. 1 (color online). Mapping of nematic patches to random resistor networks. (a) Configurations of nematic patches generated by the random field Ising model. (b) Corresponding resistor network, modeling local anisotropic conduction in each nematic patch. Solid lines are small resistors, and dotted lines are large ones. 
bicity $[6,16]$. Nematics can also be aligned by an external magnetic field due to diamagnetic anisotropy, in which case $h \propto o\left(H^{2}\right)$ [17].

The macroscopic resistance anisotropy of a nematic $[6,18,19]$ transforms under rotations in the same way as the orientational order parameter $m=(1 / N) \sum_{i} \sigma_{i}$ and is a natural candidate for measuring nematic order. The (normalized) resistance anisotropy is $R_{a} \equiv[(r+1) /$ $(r-1)]\left[\left(R_{x x}-R_{y y}\right) /\left(R_{x x}+R_{y y}\right)\right]$, where $r \equiv R_{x x}(m \rightarrow 1) /$ $R_{y y}(m \rightarrow 1)$ is the ratio of the extremal macroscopic resistances in the fully oriented state. To obtain the transport properties, we map each pattern of local nematic orientations generated by Monte Carlo simulations of the RFIM [see Fig. 1(a)] to a resistor network [see Fig. 1(b)] which models the local anisotropic transport in each nematic patch. Each patch (Ising pseudospin) becomes one node in the resistor network, with four surrounding resistors determined by the nematic orientation. For a "vertical" nematic patch $(\sigma=+1)$, we assign the resistors to the "north" and "south" to be small, $R_{\text {small }}$, while the resistors to the "east" and "west" are large, $R_{\text {large }}=r R_{\text {small }}$. For a "horizontal" nematic patch $(\sigma=-1)$, these assignments are reversed. When all patches are fully oriented so that $m \rightarrow 1$ (i.e., all nematic patches are vertical), the macroscopic resistance anisotropy saturates, $R_{a} \rightarrow 1$. More generally, in the thermodynamic limit, $\left\langle R_{a}\right\rangle=m F(m, T, r)$, where $F$ is an even function of $m$. Remarkably, as shown below, $F=1$ to a very good approximation and, under a wide range of circumstances, $\left\langle R_{a}\right\rangle \approx m$ throughout the entire range of $m$.

We use a Glauber update method to generate configurations of the RFIM, with periodic boundary conditions on the pseudospin lattice. The details of the algorithm are contained in Ref. [20]. We then calculate the resistance anisotropy $R_{a}$ of the corresponding resistor network by the following method: For $R_{x x}$, we assign a uniform applied voltage to every site at the far left end of the lattice and a uniform ground to the far right end of the lattice, with open voltage boundary conditions in the $y$ direction. We then apply the bond propagation algorithm [21] to reduce the network to a single resistor equivalent to the macroscopic $R_{x x} . R_{y y}$ is similarly calculated from the same starting resistor network but with boundary conditions appropriate for $R_{y y}$. Results shown are typical for the stated disorder strengths, although the exact shape can vary slightly with the particular disorder pattern.

We first present results at zero temperature, which exhibits the nonequilibrium behavior associated with hysteresis. Figure 2 shows a simulation of the RFIM for a system of size $L=100 \times 100$ at zero temperature. We present results for which the field $h$ is incremented at a sweep rate $\Omega=J / N$, where $N=L \times L$ is the system size and $h=\Omega t$. In Fig. 2(a), we show a hysteresis loop for the resistance anisotropy $R_{a}$ vs the symmetry-breaking field $h$, starting from the fully oriented state at $h=-\infty$.

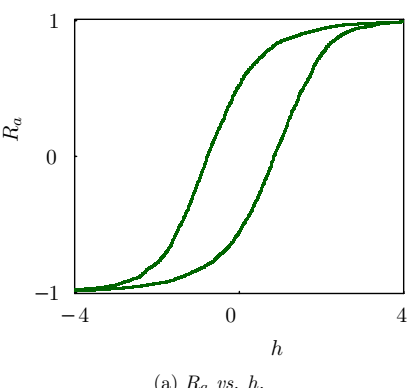

(a) $R_{a} v s . h$.

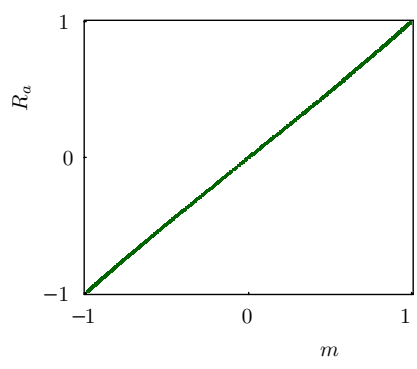

(b) $R_{a}$ vs. $m$
FIG. 2 (color online). Hysteresis and comparison of the macroscopic resistance anisotropy to the orientational order for size $L \times L=100 \times 100$, disorder strength $\Delta=3 J$, temperature $T=0$, microscopic resistance anisotropy $r=2$, and external field sweep rate $\Omega=J / N$, where $N=L \times L$. (a) Hysteresis of the resistance anisotropy $R_{a}$ (see text) vs the symmetry-breaking field $h$. (b) Resistance anisotropy $R_{a}$ vs orientational order parameter $m$. The resistance anisotropy is a remarkably good indicator of orientational order.

Figure 2(b) plots $R_{a}$ vs $m$ through one cycle of the hysteresis loop. The relationship is remarkably linear, and the macroscopic resistance anisotropy $R_{a}$ shows precisely the same hysteretic behavior as $m$. This linear relation throughout the cycle is surprising; for a given magnetization $m$ not too close to 1 , there is a range of values possible for the macroscopic resistance anisotropy, since the resistance depends not only on the relative concentration of "up" and "down" resistor nodes but also on their spatial arrangement. In fact, for $m=0, R_{a}$ can take values between $\pm\left(r^{2}-1\right) /\left(r^{2}+6 r+1\right)$. However, for typical configurations generated by the RFIM, the main contribution to $R_{a}$ is controlled by the number of up and down nodes, with their spatial relation being a small effect which goes to zero as the system size $L \rightarrow \infty$ or as $r \rightarrow 1$ [22]. (We do observe variations in $R_{a}$ between configurations with the same magnitude of $m$ at finite temperature or for small system sizes.) The fact that $\left\langle R_{a}\right\rangle \approx m$ over a wide range of parameters means that noise and hysteresis in this measurable property can be used to detect the electronic nematic.

Hysteresis subloops can be used to determine qualitatively the relative importance of interactions and disorder. Figure 3 shows the behavior of subloops in the hysteresis curve of the orientational order $m$ vs orienting field $h$. Starting from zero field and a thermally disordered configuration, the field is swept up along path 1, then switched back along path 2 to take subloop $A$ before continuing along path 3 . Then path 4 begins a second subloop, within which path 5 begins subloop $B$, before continuing to raise the field through path 6 until $m$ has saturated, $m \rightarrow 1$.

Notice that the subloops close and also that, once a subloop has closed, continuing to raise the field does not disturb the structure of the outer loop. This is indicative of return-point memory, a characteristic of the RFIM [23]. Additionally, subloops $A$ and $B$ in Fig. 3 are a comparison between the same two field strengths. The fact that the two 


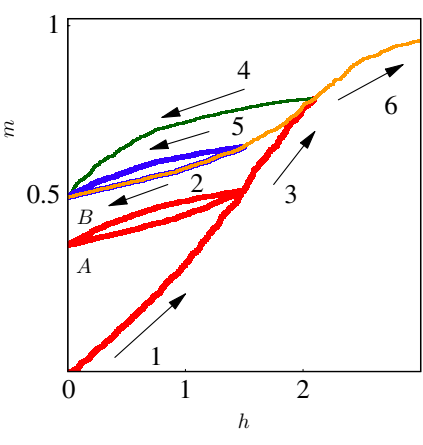

(a) Hysteresis

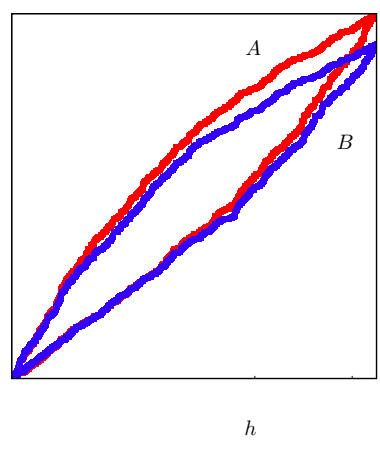

(b) Subloops
FIG. 3 (color online). (a) Field ramp-up from a disordered configuration at $T=0$, with hysteresis subloops, for system size $L \times L=100 \times 100$ and disorder strength $\Delta=3 J$. The arrows indicate the order in which the external field sweep is taken. Subloops $A$ and $B$ are both taken between the same extremal orienting fields $h=1.5 \mathrm{~J}$ and $h=2.1 \mathrm{~J}$ but with different histories. (b) Incongruency of subloops $A$ and $B$, due to the presence of interactions. Here the orientational order parameter has been shifted to compare the shape of the two subloops. Subloop $A$, executed at lower $m$, has a higher slope than subloop $B$, executed at higher $m$.

are incongruent as shown in Fig. 3(b) indicates that interactions are present and possibly even avalanches, i.e., that the hysteresis is not simply a linear superposition of elementary hysteresis loops of independent grains as in the Preisach model [24-26]. Here we have used a disorder strength of $\Delta=3 J>\Delta_{\text {crit }}^{3 \mathrm{D}}=2.16 J$. At lower disorder, the subloops become narrower and difficult to resolve. Also, as the temperature is increased, thermal disorder means that subloops become narrower and no longer close precisely.

Recent magnetization measurements by Panagopoulos et al. $[9,10]$ on $\mathrm{La}_{2-x} \mathrm{Sr}_{x} \mathrm{CuO}_{4}$ (LSCO) reveal hysteretic behavior reminiscent of the RFIM. Although some of the results look very similar to our Fig. 3, in fact our model does not explicitly include the magnetic moments measured in this experiment. However, the small magnitude of the measured magnetization may be consistent with ferromagnetic moments arising at defects in the local striped antiferromagnetic order of a nematic patch.

We now discuss equilibrium fluctuations. In confined geometries such as nanowires and dots, this model can exhibit telegraph noise in $R_{a}$ due to thermal fluctuations of large correlated clusters. Recent transport experiments on underdoped yttrium barium copper oxide (YBCO) nanowires by Bonetti et al. [8] reveal telegraphlike noise in the pseudogap regime. Using a YBCO nanowire of size $250 \mathrm{~nm} \times 500 \mathrm{~nm}$, they found that a time trace of the resistance at constant temperature $T=100 \mathrm{~K}$ shows telegraphlike fluctuations of magnitude $0.25 \%$, on time scales on the order of 50 seconds. These large scale, slow switches can be understood within our model as thermal fluctuations of a single correlated cluster of nematic patches. Individual nematic patches thermally fluctuating between two orientations also produce noise, but at higher frequency, and with smaller effect on the macroscopic resistance. By comparing the magnitude of the smallest typical resistance change to the resistance change at a telegraph noise event, one can estimate that the correlated cluster in the nanowire contains at least 5-6 nematic patches.

The nanowire corresponds to a rather small system size when mapped to the RFIM. Neutron scattering experiments on the incommensurate peaks of underdoped YBCO indicate a coherence length of roughly $40 \mathrm{~nm}$. If we take this as an estimate of the size of one nematic patch (mapped to a single pseudospin in the RFIM), the nanowire is about $6 \times 12$ patches wide, and the effect of flipping a correlated cluster of pseudospins can dominate the response. In Fig. 4(a), we show a time series of $R_{x x}$ in thermal equilibrium $(h=0)$ for a small system of size $L \times L=$ $6 \times 6$ at finite temperature with disorder strength $\Delta=2 J$. We take $r=2$ as a representative value, being larger than but of order 1. In fact, in the experiments of Ref. [6], the lattice orthorhombicity acts as an orienting field $h$, and the measured range of resistivity anisotropy $\rho_{a} / \rho_{b} \approx 1.2-2.6$ may be taken as a rough lower bound on $r$. Notice the sizable thermal fluctuations in the macroscopic resistance. The high frequency noise is due to the thermal fluctuations of a single Ising pseudospin. The lower frequency telegraph noise, in which the resistance changes dramatically, is due to the correlated fluctuations of a cluster of pseudospins, in our case a cluster of 4 pseudospins. The histogram in Fig. 4(b) shows that the system switches between two main states.

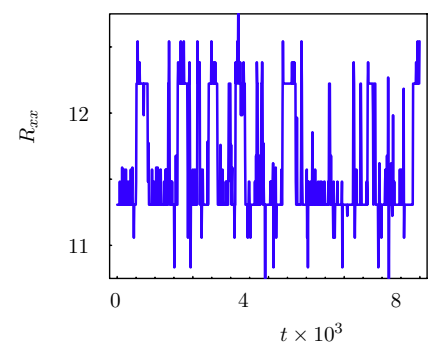

(a) $R_{x x} v s$. time

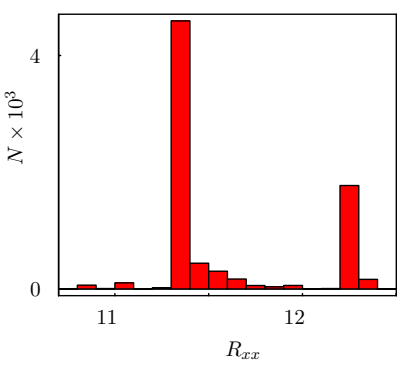

(b) Histogram
FIG. 4 (color online). (a) Telegraph noise in a confined geometry. Resistance $R_{x x}$ vs time of a small system of size $L \times$ $L=6 \times 6$ with disorder strength $\Delta=2 J$, at temperature $T=$ $0.5 \mathrm{~J}$, in zero applied field, $H=0$. An initial random state is allowed to thermalize for 10000 Monte Carlo steps before measurements are taken. The local microscopic conductivity anisotropy in the resistor network is $r=2$. Small fluctuations are due to a single nematic patch fluctuating. Large switches are due to the thermal fluctuations of a single correlated cluster of nematic patches. (b) Histogram of the time series. $N$ is the number of occurrences of a particular resistance. There are two main states, one for which the correlated cluster is up, and the other for which it is down. 
In general, correlated clusters will thermally switch with a time scale which rapidly increases with their size. Confined geometries such as the nanowire are more likely to have a system-spanning cluster which causes slow, large scale noise. For larger system sizes, the histogram becomes smoother and can have multiple peaks. For systems larger than the Imry-Ma correlation length, large scale switches are highly unlikely. A probability distribution of size of cluster vs frequency can be obtained from a histogram of the differential resistance, in which large scale switches will always be relegated to the tails of the distribution.

A further way to test for nematic patches is to measure cross correlations between the thermal noise in $R_{x x}$ and $R_{y y}$ as a function of time [27]. For example, resistance fluctuations due to fluctuation superconductivity would cause correlated fluctuations of $R_{x x}$ and $R_{y y}$, while nematic fluctuations would cause anticorrelated fluctuations.

This general method of connecting local anisotropic properties of an electronic nematic to macroscopic behavior can be extended to many experimental measurements. For example, applying an in-plane magnetic field should drive the fourfold symmetric incommensurate spin peaks in neutron scattering into a twofold symmetric pattern, in a hysteretic manner as the field is rotated from one $\mathrm{Cu}-\mathrm{O}$ direction to the other. Superfluid density anisotropy should also display hysteresis with in-plane field orientation. Furthermore, the presence of correlated clusters in the RFIM has implications for STM. Whereas small correlated clusters have fast switching dynamics, large clusters are much slower [24], so that different size clusters have different local power spectra. STM can be used to do scanning noise spectroscopy [28], by measuring the power spectrum as a function of position, in order to produce a spatial map of correlated nematic clusters.

In conclusion, we have mapped the electron nematic in a host crystal to the random field Ising model. Using a further mapping to a random resistor network, we have predicted new ways to detect the electron nematic in disordered systems. We have demonstrated that the macroscopic resistance anisotropy is a good measure of orientational order and is expected to display hysteresis and thermal noise characteristic of the random field Ising model. Recent experiments on noise in YBCO nanowires and hysteresis in LSCO exhibit behavior reminiscent of this model.

We thank T. Bonetti, D. Caplan, D. Van Harlingen, C. Panagopoulos, and M. Weissman for many illuminating conversations which motivated this work and for sharing their unpublished work, and we thank T. Datta and J. C. Davis for helpful discussions. This work was supported in part through The Purdue Research Foundation (E. W. C.) and by NSF Grants No. DMR 03-25939 and No. DMR 0314279 at UIUC, No. PHY 99-07949 at the KITP-UCSB (K. A.D.), No. DMR 04-42537 at UIUC (E.F.), and No. DMR-04-21960 at Stanford and UCLA (S. A. K.).
[1] S. A. Kivelson, E. Fradkin, and V. J. Emery, Nature (London) 393, 550 (1998).

[2] S. Chakravarty, R. B. Laughlin, D. K. Morr, and C. Nayak, Phys. Rev. B 63, 094503 (2001).

[3] S. Kivelson et al., Rev. Mod. Phys. 75, 1201 (2003).

[4] E. W. Carlson, V. J. Emery, S. A. Kivelson, and D. Orgad, in The Physics of Superconductors, edited by J. Ketterson and K. Benneman (Springer-Verlag, Berlin, 2004), Vol. II.

[5] C. M. Varma, Phys. Rev. B 55, 14554 (1997).

[6] Y. Ando, K. Segawa, S. Komiya, and A. N. Lavrov, Phys. Rev. Lett. 88, 137005 (2002).

[7] T. Nattermann, in Spin Glasses and Random Fields, edited by A. Young (World Scientific, Singapore, 1998).

[8] J. A. Bonetti, D. S. Caplan, D. J. Van Harlingen, and M. B. Weissman, Phys. Rev. Lett. 93, 087002 (2004).

[9] C. Panagopoulos, M. Majoros, T. Nishizaki, and H. Iwasaki, cond-mat/0412570.

[10] C. Panagopoulos, M. Majoros, and A. Petrović, Phys. Rev. B 69, 144508 (2004).

[11] Y. Imry and S. K. Ma, Phys. Rev. Lett. 35, 1399 (1975).

[12] O. Zachar and I. Zaliznyak, Phys. Rev. Lett. 91, 036401 (2003).

[13] A. Abanov, V. Kalatsky, V.L. Pokrovsky, and W. M. Saslow, Phys. Rev. B 51, 1023 (1995).

[14] The effect of different sized patches may be subsumed into randomness in the coupling $J$, which will not change the qualitative behavior of our model, although it will change the results quantitatively for small systems.

[15] C. Reichhardt, C. J. Olson Reichhardt, and A. R. Bishop, Europhys. Lett. 72, 444 (2005).

[16] Ando et al. [6] report the observation of a temperaturedependent increase of the resistance anisotropy in very underdoped LSCO and YBCO. In both systems, the lattice is orthorhombic, which acts as a stabilizing symmetrybreaking field, thus overcoming the overwhelming effects of disorder in these quasi-2D systems.

[17] P. Chaikin and T. Lubensky, Principles of Condensed Matter Physics (Cambridge University Press, Cambridge, England, 1995).

[18] E. Fradkin and S. A. Kivelson, Phys. Rev. B 59, 8065 (1999).

[19] K. B. Cooper et al., Solid State Commun. 119, 89 (2001).

[20] R. A. White, A. Travesset, and K. A. Dahmen, Thermal Effects on Crackling Noise (to be published).

[21] D. J. Frank and C. J. Lobb, Phys. Rev. B 37, 302 (1988).

[22] E. W. Carlson, K. A. Dahmen, E. Fradkin, and S. A. Kivelson (to be published).

[23] J. P. Sethna et al., Phys. Rev. Lett. 70, 3347 (1993).

[24] J. P. Sethna, K. A. Dahmen, and O. Perković, in Science of Hysteresis, edited by I.D. Mayergoyz and G. Bertotti (Academic, London, 2004).

[25] M. P. Lilly, P. T. Finley, and R. B. Hallock, Phys. Rev. Lett. 71, 4186 (1993).

[26] I. D. Mayergoyz, Mathematical Models of Hysteresis (Springer-Verlag, Berlin, 1991).

[27] R. D. Black, P. J. Restle, and M. B. Weissman, Phys. Rev. Lett. 51, 1476 (1983).

[28] M.E. Welland and R. H. Koch, Appl. Phys. Lett. 48, 724 (1986). 